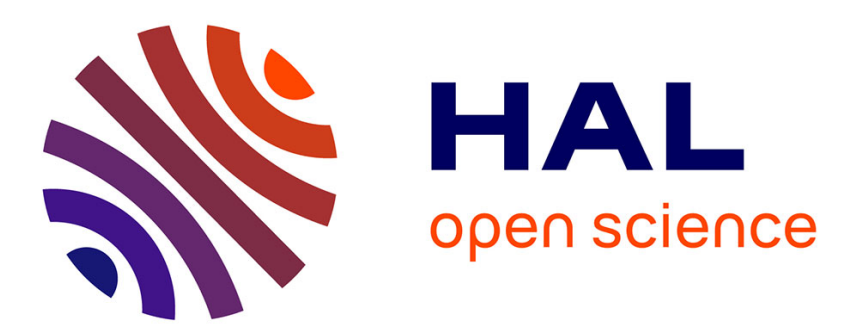

\title{
Les systèmes: Un enjeu épistémologique de la géographie des lumières / Systems : Philosophical consequences of enlightenment geography
}

\author{
Isabelle Laboulais
}

\section{- To cite this version:}

Isabelle Laboulais. Les systèmes: Un enjeu épistémologique de la géographie des lumières / Systems : Philosophical consequences of enlightenment geography. Revue d'Histoire des Sciences, 2006,59 (1), pp.97-125. 10.3406/rhs.2006.2268 . hal-02932793

\section{HAL Id: hal-02932793 \\ https://hal.science/hal-02932793}

Submitted on 7 Sep 2020

HAL is a multi-disciplinary open access archive for the deposit and dissemination of scientific research documents, whether they are published or not. The documents may come from teaching and research institutions in France or abroad, or from public or private research centers.
L'archive ouverte pluridisciplinaire HAL, est destinée au dépôt et à la diffusion de documents scientifiques de niveau recherche, publiés ou non, émanant des établissements d'enseignement et de recherche français ou étrangers, des laboratoires publics ou privés. 


\title{
Les systèmes : Un enjeu épistémologique de la géographie des lumières
}

\author{
Isabelle LABOULAIS *
}

\begin{abstract}
Résumé : Étudier la manière dont les géographes français des lumières ont eu recours aux systèmes constitue un moyen $d^{\prime}$ interroger la capacité de la géographie à produire des règles d'appréhension du monde, un moyen aussi de se demander si les systèmes peuvent jouer ce rôle et à quelles conditions ils le peuvent. Au XVIII ${ }^{\mathrm{e}}$ siècle, à une période où les observations font encore largement défaut pour tracer les contours des continents et décrire la forme des reliefs, les géographes ont mobilisé les systèmes de différentes manières. De façon assez unanime, les cartographes regardaient le système comme l'agencement des données grâce auquel ils étaient parvenus à construire leur carte. En revanche, de manière plus polémique, dans le domaine de la géographie physique, certains géographes ont utilisé les systèmes pour tenter de poser les principes qui, à leurs yeux, pouvaient régir les formes de la terre. Cette méthode a suscité débats et controverses dans la deuxième moitié du XVIIII ${ }^{\mathrm{e}}$ siècle, débats et controverses qui éclairent à la fois les usages des systèmes et le statut de la géographie au Siècle des lumières.
\end{abstract}

Mots-Clés : système ; géographie physique; cartographie ; Académie royale des sciences.

\begin{abstract}
Summary: Studying the way French geographers of the enlightenment made use of systems offers a way to question the ability the field of geography had to produce rules for understanding the world. It also questions whether systems could in fact play this role and under what conditions. In the eighteenth century, at a moment when detailed observations were not available to trace the outlines of continents or to describe their relief, geographers drew on systems in a variety of ways. Almost unanimously cartographers considered systems as the ordering of information that allowed them then to construct maps. More polemically, however, in physical geography, some used systems as a way of establishing the principles that in their view underlay the earth's varied forms. This method generated debates and controversy in the second half of the eighteenth century ; these debates reveal not only the way systems were used but also the status of geography during the enlightenment.
\end{abstract}

Keywords : system ; physical geography ; cartography ; The Royal academy of sciences.

* Isabelle Laboulais, Palais universitaire, Institut d'histoire moderne, Université Marc BlochStrasbourg 2, 9, place de I'Université,67084 Strasbourg. isabelle.laboulais@wanadoo.fr 
Les systèmes sont des outils de la pensée, des constructions théoriques qui permettent l'étude et la connaissance d'un objet donné. Toutefois, dans le contexte anticartésien qui promeut l'expérimentation et le raisonnement inductif, l'esprit de système se voit associé à la métaphysique du $X V \|^{e}$ siècle et est ainsi condamné par les hommes des lumières. Le physicien n'a plus pour mission d'expliquer le mécanisme de l'univers mais de montrer les relations qui unissent les différents éléments qui le constituent. Cependant, les hommes des lumières ne peuvent se satisfaire d'une accumulation de faits épars, ils aspirent à l'établissement de régularités, voire de généralisations. Or, dans cette perspective, les systèmes apparaissent comme une garantie contre les dangers d'un empirisme trop étroit et les risques d'une vision atomisée de la nature. Comme l'a relevé Ernst Cassirer, "Posséder le système sans être possédé par lui [...]. C'est une nouvelle direction de recherche et pour ainsi dire un nouveau tempérament de chercheur qui se fait jour exigeant d'être reconnu, justifié dans son style propre et validé dans sa méthode ${ }^{1} »$.

Les géographes français du Siècle des lumières ont, eux aussi, été concernés par l'ambivalence des systèmes. Pour eux, les systèmes offrent un moyen de rendre compte d'un phénomène spatial ; souvent, ils leur permettent de dépasser le niveau de la description pour tendre vers celui de l'explication, d'articuler des faits pour expliquer leur agencement et rendre intelligibles des données de terrain disparates. Étudier le recours des géographes aux systèmes consiste donc à s'interroger sur la capacité de la géographie à produire des règles d'appréhension du monde, cela invite aussi à se demander si les systèmes peuvent jouer ce rôle et à quelles conditions.

Alors que la géographie jouit d'un statut épistémologique complexe, alors qu'elle doit s'imposer face à des voisinages disciplinaires conquérants, on peut se demander si les systèmes n'ont pas été, au Siècle des lumières, une manière de "faire science ", c'est-à-dire une façon pour les géographes de s'affirmer au sein de la communauté savante, un moyen aussi de légitimer leurs propres actes en tentant de reconstruire la géographie selon le modèle des sciences exactes.

1 - Ernst Cassirer, La Philosophie des lumières (Paris : Fayard, 1990 [1 $1^{\text {re }}$ éd., 1966]), 103. "Nous ne pouvons commencer par des hypothèses générales sur la nature des choses pour en déduire par la suite la connaissance des effets particuliers ; nous devons au contraire mettre en tête de notre recherche la connaissance telle qu'elle nous est donnée dans l'observation directe pour tenter de parvenir ensuite, en nous élevant progressivement, jusqu'aux premières causes et aux éléments les plus simples du cours des choses. À l'idéal de la déduction s'oppose ainsi I'idéal de I'analyse. » (Ibid., 81-82); on consultera également Jean Erhard, L'Idée de nature en France à l'aube des lumières (Paris: Flammarion, 1970), 23-152 et Georges Gusdorf, Les Sciences humaines et la pensée occidentale, t. 4, Les Principes de la pensée au Siècle des lumières (Paris : Payot, 1971), chap. III, V. 


\section{Le rôle des systèmes dans la construction d'une carte}

De manière assez courante, les géographes du XVIII ${ }^{\mathrm{e}}$ siècle parlent de "système géographique » pour désigner les moyens grâce auxquels ils ont réuni des données d'origines différentes, la façon dont ils les ont critiquées, comparées et articulées pour parvenir à cartographier un espace le plus précisément possible. À ce stade, ils ne cherchent pas à proposer de principes explicatifs mais ils s'efforcent de construire une représentation textuelle et cartographique rigoureuse. Ainsi, lorsqu'en 1774 Didier Robert de Vaugondy (1723-1786) publie une carte qu'il intitule " Nouveau système géographique », il entend rendre compte de la méthode qu'il a mise en œuvre pour combiner des éléments congruents lorsqu'il a dressé sa carte du littoral du Nord-Ouest de I'Amérique et du Nord-Est de l'Asie (voir illustration 1). Pour mesurer les enjeux du recours au mot " système » dans le titre de cette carte, il faut resituer le contexte de sa publication.

Depuis le début du $x \mathrm{VIII}^{\mathrm{e}}$ siècle, cette partie du monde est sujette à de nombreuses conjectures : la Californie, le Japon et le Kamtchatka sont mal identifiés; et le détroit d'Anian, par lequel à la fin du XvI ${ }^{e}$ siècle Abraham Ortelius a symbolisé le fameux passage du Nord-Ouest censé relier le Nord de I'Asie avec le Nord de I'Amérique, suscite encore de nombreuses questions ${ }^{2}$. Ainsi, sur la carte d'Amérique du Nord qu'il publie en 1695, Guillaume Delisle (1675-1726) laisse-t-il en blanc le tracé des côtes nord-ouest du continent, considérant qu'il existe alors trop d'incertitudes pour les cartographier. II se contente $d^{\prime}$ 'esquisser le détroit d'Anian pour en faire la jonction entre la mer de l'ouest et la mer du sud ${ }^{3}$. Par la suite, de nombreux géographes ont construit d'autres hypothèses pour cartographier cette partie du monde, leurs hypothèses sont fondées sur les résultats des nombreuses expéditions maritimes financées par les puissances européennes. Parmi elles se trouve la relation de l'amiral de Fonte, un amiral espagnol qui se serait rendu au nord-ouest du continent américain en 1640. Ce texte n'est pourtant diffusé en France qu'en 1750, par I'intermédiaire de I'astronome Joseph-Nicolas Delisle (1688-1768), frère cadet du géographe Guillaume Delisle, qui ramène ce document de Saint-Pétersbourg où il a séjourné pendant vingt et un ans, le temps notamment pour lui de fonder I'observatoire. De retour à Paris, Delisle transmet donc à Philippe Buache (1700-1773) la relation du voyage de l'amiral de Fonte, qui n'est en fait qu'une lettre apocryphe ${ }^{4}$. De son côté, l'astronome publie un traité intitulé Nouvelles cartes des décou-

2 - Lucie Lagarde, Le passage du Nord-Ouest et la mer de I'ouest dans la cartographie française du XVIII ${ }^{\mathrm{e}}$ siècle : Contribution à l'étude de I'œuvre des Delisle et Buache, Imago Mundi, 41 (1989), 19-43.

3 - Annick Foucrier, La preuve par les coquillages bleus : La Californie (XVI ${ }^{\mathrm{e}}-\mathrm{XVIII}{ }^{\mathrm{e}}$ siècle), île ou péninsule?, Genèses, 40 (2000), 33-52, voir en particulier 47-51.

4 - Cette lettre a été publiée à Londres en avril et juin 1708 dans le périodique Monthly miscellany or memoirs for the curious. L'auteur de ce texte est probablement l'éditeur du périodique, James Petiver. Voir Lagarde, op. cit. in n. 2. 
vertes de l'amiral de Fonte, et autres navigateurs espagnols, portugois, anglois, hollandois, françois [et] russes, dans les mers septentrionales, avec leur explication : qui comprend, I'histoire des voyages, tant par terre que par mer, dans la partie septentrionale de la terre, les routes de navigation. Dans cet opuscule de 71 pages, il reprend plusieurs des hypothèses cartographiques de son frère, y compris son " système » de 1695 (voir illustration 1), et entend préciser la connaissance de ce territoire. La comparaison des cartes des Delisle permet de mesurer que les blancs laissés par Guillaume ont été largement comblés par son frère. Sur les cartes que Joseph-Nicolas publie en 1752 (voir illustrations 2 et 3), les contours de la mer de l'ouest sont désormais établis, le réseau des lacs du continent nord-américain est en place et le détroit d'Anian devient, d'après les informations de l'amiral de Fonte, celui qui permet d'accéder au réseau hydrographique ouvrant l'accès à la baie d'Hudson.

La même année, le 9 août 1752, Buache présente à son tour à I'Académie royale des sciences ses «Considérations géographiques et physiques sur ce que la carte des nouvelles découvertes offre de plus particulier 5 ». Ce mémoire expose les principes suivant lesquels il a construit sa carte - Carte des découvertes de l'amiral de Fonte selon la carte angloise donnée par l'écrivain du vaisseau la Californie dans son voyage à la baie d'Hudson avec les terres vues et reconnues par les Russes et une comparaison du résultat des cartes du XVI et du XVII siècle au sujet du détroit d'Anian (voir illustration 4) - et la manière dont il a intégré les données de cette relation pour cartographier « la figure des païs compris entre le Kamtchatka et le Nord-Ouest de l'Amérique ${ }^{6} »$.

Dans ce mémoire, Buache parle de "système » pour désigner la construction cartographique à laquelle il est parvenu. II s'agit bien alors d'un agencement des données qui le conduit à compléter la carte de Delisle en précisant notamment la situation de la " mer de l'ouest », en considérant, lui aussi, le détroit d'Anian comme un moyen d'accéder à la baie d'Hudson. II reste cependant plus prudent que JeanNicolas Delisle et ne cartographie pas l'ensemble des côtes nordaméricaines. Pour la circonstance, Buache joint à son mémoire I'«Essai d'une carte de la mer de l'ouest » dessinée par Guillaume

5 - Isabelle Laboulais, Le système de Buache, une " nouvelle façon de considérer notre globe » et de combler les blancs de la carte au milieu du XVIII ${ }^{\mathrm{e}}$ siècle, in Combler les blancs de la carte : Modalités et enjeux de la construction du savoir cartographique (XVI-XIX siècle), sous la dir. d'Isabelle Laboulais (Strasbourg : Presses univ. de Strasbourg, 2004), 93-115.

6 - Philippe Buache, "Considérations géographiques et physiques sur ce que la carte des nouvelles découvertes offre de plus particulier ", ms., pochette 9 août 1752 (Arch. de I'Académie royale des sciences), 1. (Toutes les citations respectent l'orthographe originale.) 


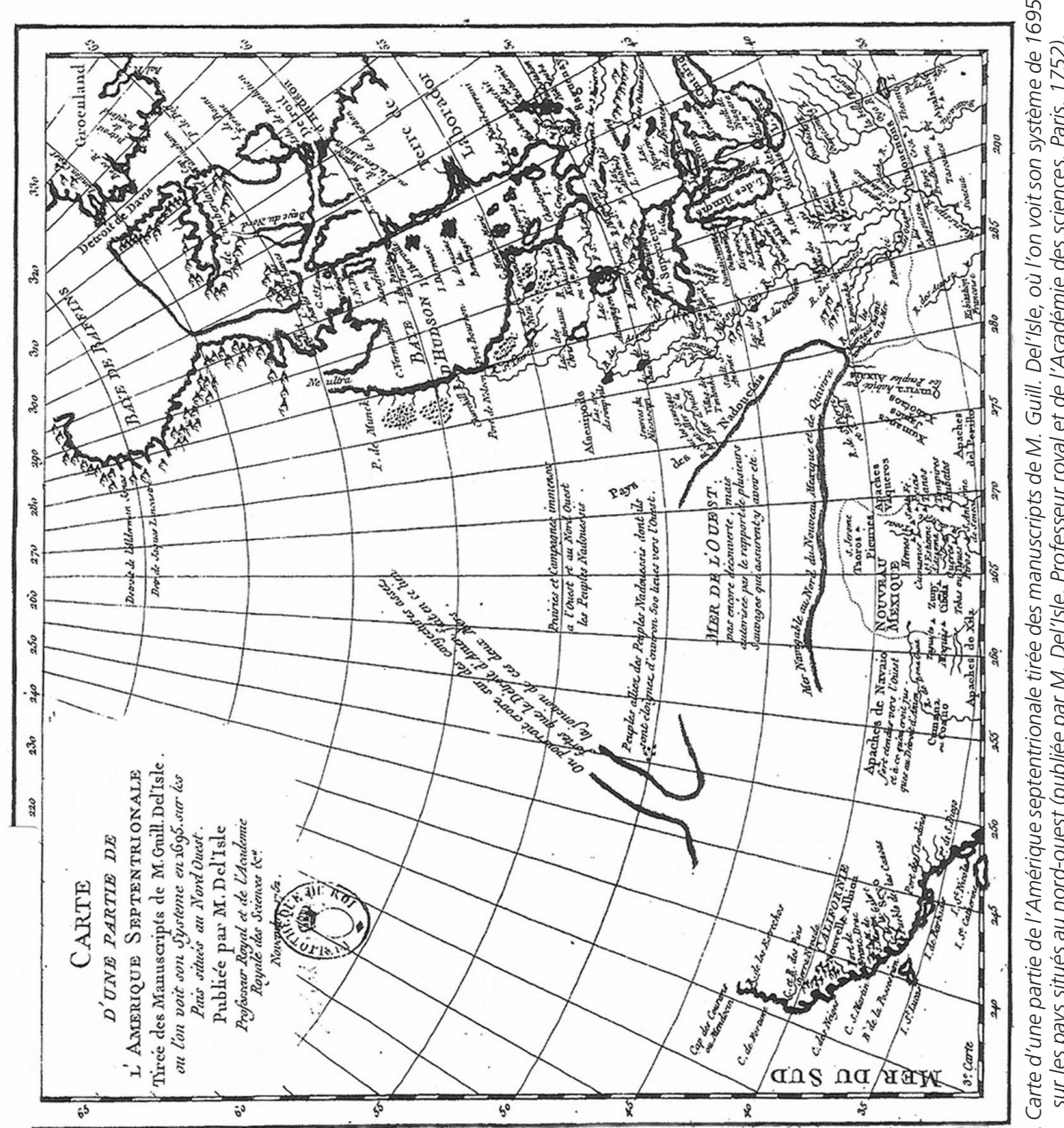




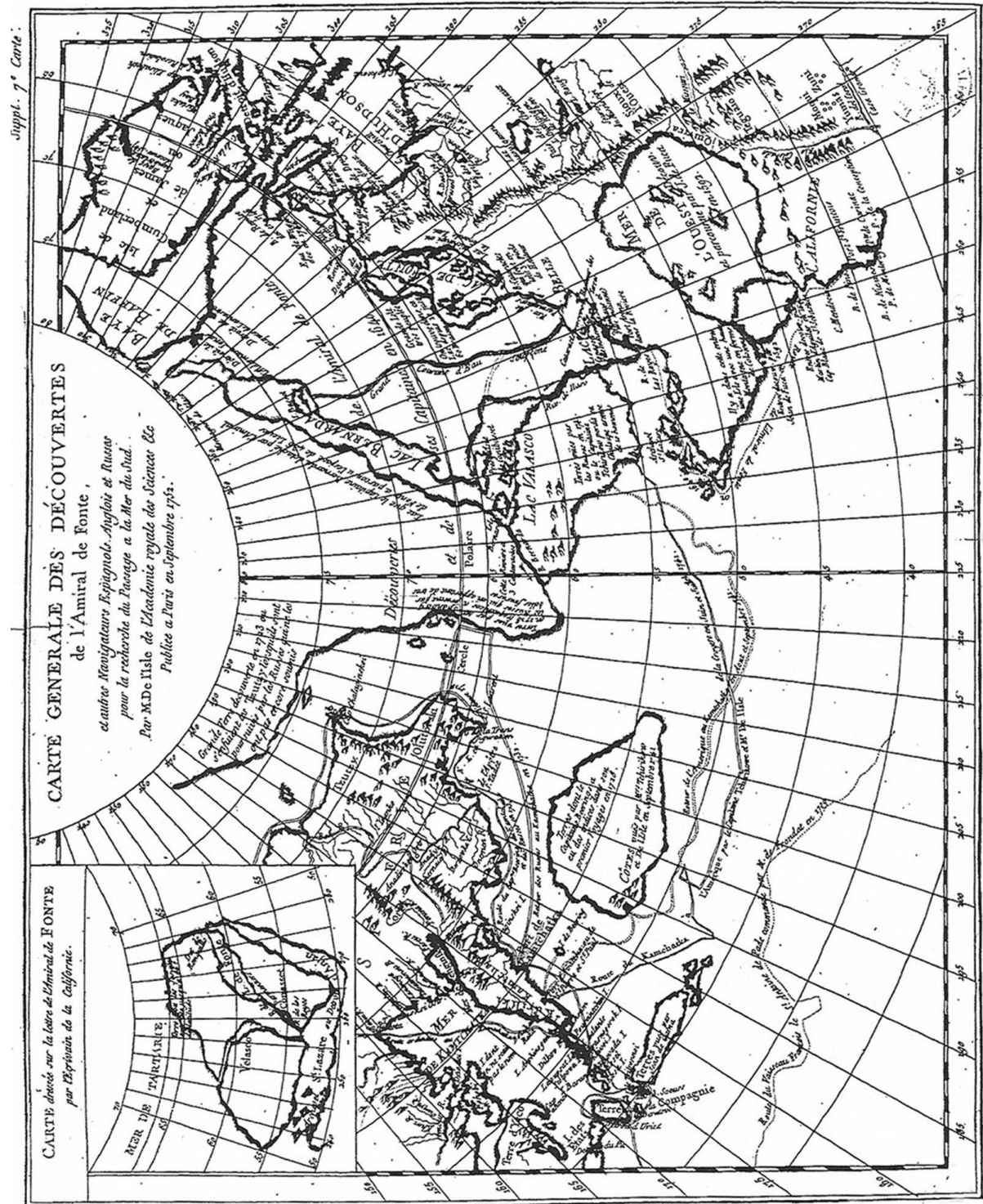




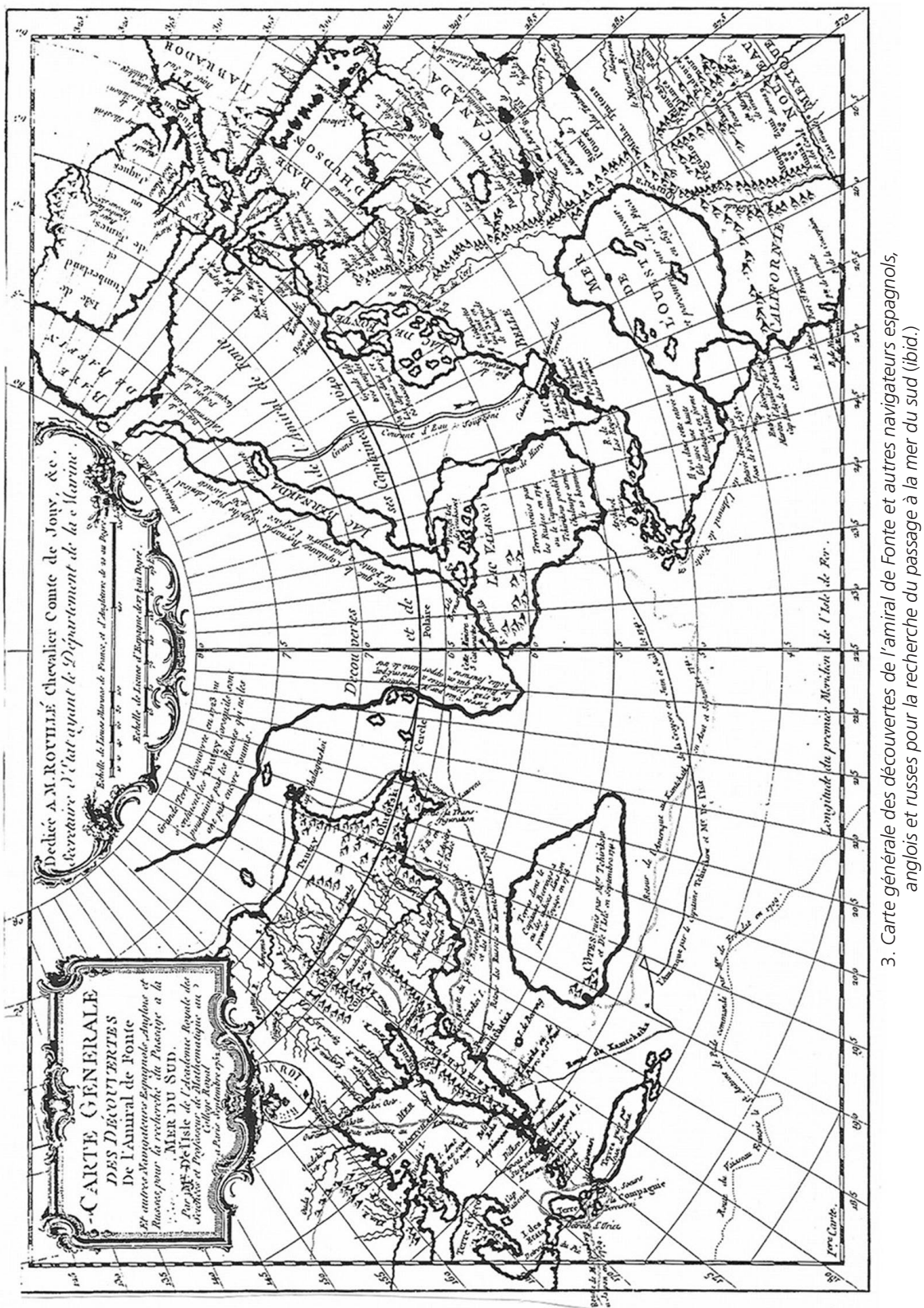




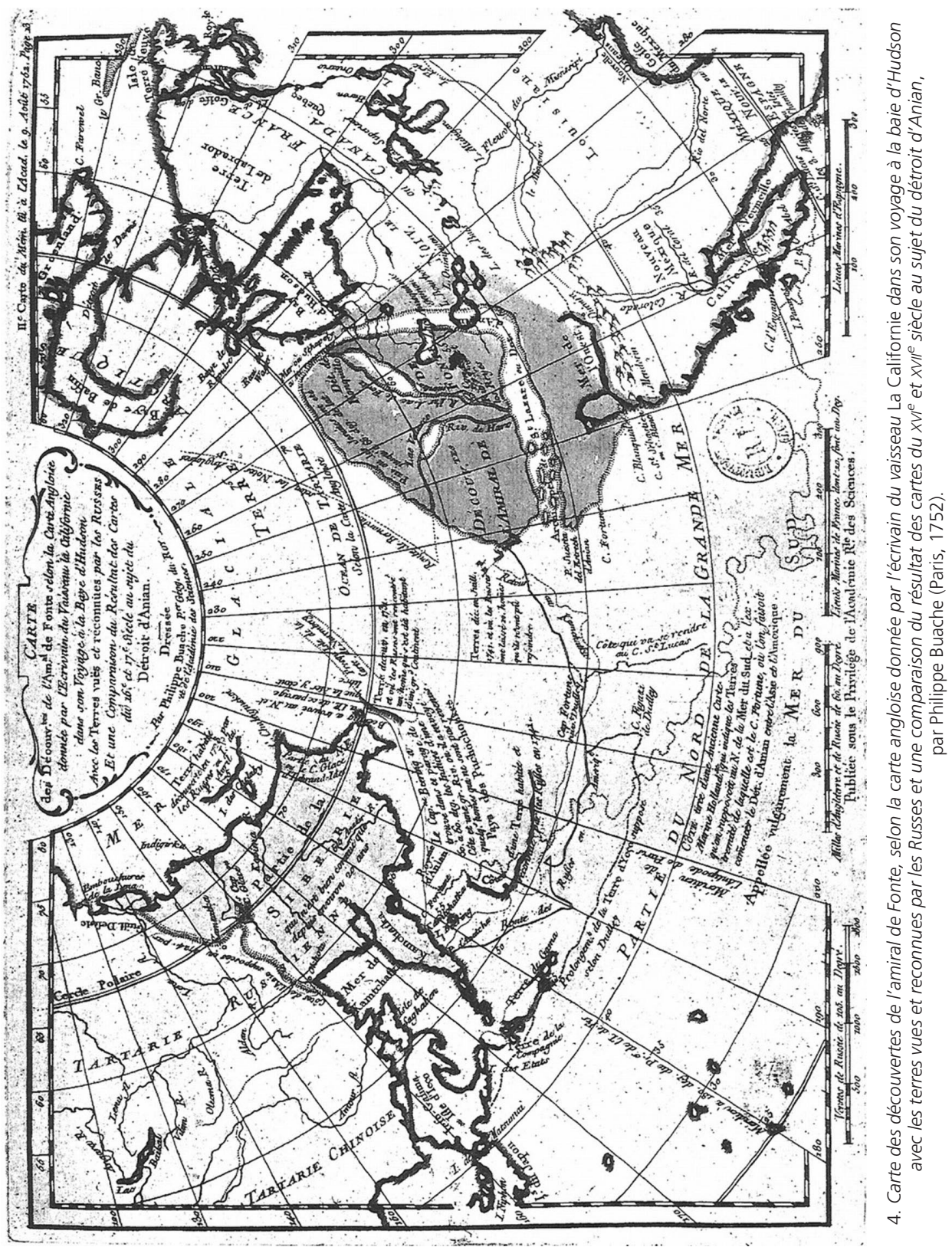


Delisle (voir illustration 5) qui vient appuyer son argumentation. Le nom du géographe, membre de la classe d'astronomie de l'Académie des sciences est ici mobilisé comme une caution, comme un vecteur de légitimation. Comme le montre l'éloge que Fontenelle a rédigé, les travaux cartographiques de Delisle étaient alors étroitement associés au souci d'exactitude, mais aussi à la mise en place d'une cartographie fiable. Lorsqu'il évoque les travaux de Delisle, publiés en 1699, Fontenelle affirme : "Plusieurs points de la Terre prenoient enfin des places qu'ils ne pouvoient plus perdre, \& auxquelles les autres devoient s'assujettir. [...] L'ouverture du siècle présent se fit donc à l'égard de la géographie par une Terre presque nouvelle que M. Delisle présenta ${ }^{7}$. »

Utiliser les travaux de Guillaume Delisle constitue donc pour Buache un moyen de légitimer son interprétation. Pourtant, I'année suivante, de Vaugondy publie une critique acerbe de la méthode et des conclusions de son confrère, ce sont ses Observations critiques sur les nouvelles découvertes de l'Amiral de la Fuente. Dans ce texte, il regarde en effet cette relation espagnole comme un faux et accuse Buache de l'utiliser seulement parce qu'elle corrobore sa conception des contours du continent américain. Buache répond à Robert de Vaugondy, qui à son tour réplique. Les positions de chacun sont exposées dans le Journal des savants puis publiées dans de petits opuscules. L'enjeu majeur à cette époque regarde la véracité supposée de la relation de l'amiral de Fonte. Cette polémique imprimée dure ainsi une année sans que la question ne soit tranchée; ce n'est finalement que vingt années plus tard, et un an après la mort de Buache, que de Vaugondy publie cette fois un « nouveau système géographique », dans lequel il expose la méthode suivant laquelle il est parvenu à concilier diverses sources d'informations pour dresser sa carte. Le titre qu'il donne à sa carte décrit d'ailleurs sa méthode : « Nouveau système géographique par lequel on concilie les anciennes connoissances sur les pays nord-ouest de l'Amérique avec les nouvelles découvertes des Russes au nord de la mer du sud ». Dans le mémoire qu'il publie en 1774 pour présenter cette carte, de Vaugondy désigne plus explicitement encore ses sources :

«Mon projet est de combiner, non seulement la partie orientale de la Sibérie, mais encore les découvertes faites par les Russes en 1741, à I'est du Kamtchatka, avec celles qui ont été attribuées à l'amiral espagnol de Fonte, faites en 1640, \& publiées par Mrs Delisle \& Buache en 1750. Je ne crois pas devoir oublier les connoissances des XVI ${ }^{\mathrm{e}}, \mathrm{XVII}^{\mathrm{e}}$ et XVIII ${ }^{\mathrm{e}}$ siècles, d'autant qu'elles paroissent pouvoir se concilier avec celles que l'on a eues depuis par les sauvages de l'Amérique, touchant

7 - Bernard Fontenelle, Éloge de M. Delisle, in Éloges des académiciens avec l'histoire de I'Académie royale des sciences en MDCXCIX avec un discours préliminaire sur l'utilité des mathématiques (La Haye : Isaac Van der Kloot, 1740), 284. 


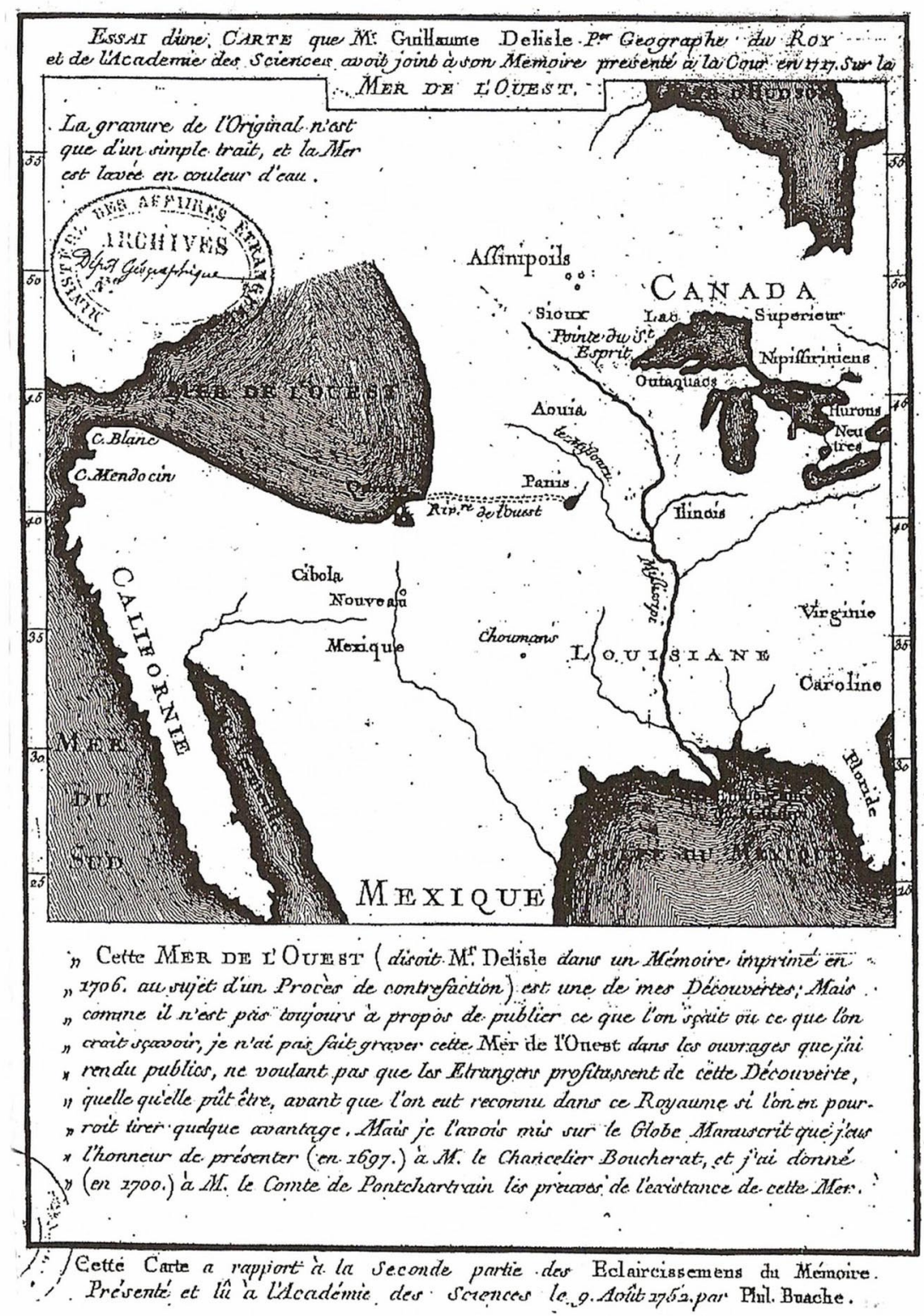

5. Essai d'une carte que M. Guillaume Delisle, premier géographe du Roy et de l'Académie des sciences avait joint à son mémoire présenté à la cour en 1727 sur la mer de l'ouest (Paris, 1752). 
les pays situés à l'Ouest du Canada \& au Nord du Nouveau-Mexique, sans prétendre vouloir donner à toutes ces connoissances le même degré $d^{\prime}$ authenticité ${ }^{8}$. »

Les sources de de Vaugondy sont donc de nature diverse ; il s'appuie à la fois sur des relations de voyages et sur des savoirs géographiques, il mêle des observations d'Européens et des observations d'indigènes, enfin il ne semble accorder aucune préférence aux découvertes les plus récentes. La cohérence de ces données, leur compatibilité avec le système ne peuvent être évaluées que par la carte; si des données s'insèrent dans le carroyage et permettent de construire des contours cohérents, elles peuvent dès lors être admises. Le système est donc ici plus cartographique que textuel. C'est le carroyage de la carte qui fonde la valeur du système. De Vaugondy note d'ailleurs :

«Pour mieux fixer l'attention dans la lecture de ce mémoire je l'accompagne d'une carte, dans laquelle je présente un système qui m'a paru plus propre que les précédens à concilier toutes ces découvertes : mais pour procéder avec ordre dans la construction de cette carte, \& dans l'examen des objets qu'il m'a fallu discuter, j'ai commencé par établir des points mixtes sur lesquels doivent porter toutes les parties du système que j'ai entrepris de développer. Ces points se réduisent à deux principaux qui sont, à l'ouest la presqu'isle du Kamtchatka, \& à l'est celle de la Californie dont il a été nécessaire de déterminer la distance réciproque en longitude ${ }^{9}$.

Pour de Vaugondy, la carte fait preuve. Son système cartographique repose donc sur deux «points mixtes »- deux positions - à partir desquels il décide d'intégrer ou non les autres données chiffrées et précises, elles aussi. Ainsi, vingt ans après la controverse qu'il avait suscitée avec Buache, de Vaugondy affine sa critique envers le voyage du fameux amiral, et plutôt que de s'arrêter sur l'existence réelle ou fantasmée de cette expédition, il insiste sur l'impossibilité pour un géographe de se satisfaire des données approximatives réunies par l'amiral de Fonte et affirme que les résultats des observations doivent être datés, au risque sinon d'être tenus à l'écart du corpus des géographes :

«Ce rapport d'un savant astronome, tel que M. Delisle, méritoit bien la confiance qu'on lui avoit donnée; mais il ne devoit cependant pas autant captiver, comme s'il eut été témoin oculaire de ces observations, ou qu'il les eut faites lui-même. Au lieu d'annoncer aussi vaguement ces observations des satellites de Jupiter, il auroit fallu les étayer de dates \& même publier les calculs qui ont dû en donner les résultats. Un point aussi intéressant, qui constate les limites d'un continent, exigeoit

8 - Didier Robert de Vaugondy, Mémoire sur les pays de l'Asie et de l'Amérique, situés au nord de la mer du sud: accompagné d'une carte intitulée: "Nouveau système géographique par lequel on concilie les anciennes connoissances sur les pays nord-ouest de l'Amérique, avec les nouvelles découvertes des Russes au nord de la mer du sud. » (Paris: Antoine Boudet, 1774), 2-3.

9 - De Vaugondy, op. cit. in n. 8, 3-4. 
que I'on en détaillât les circonstances, autrement l'on aura toujours un juste sujet de défiance sur une pareille détermination ${ }^{10}$. »

Le système est donc ici un arrangement, une manière de combiner les données sur la carte. II doit permettre de construire une représentation cohérente du monde. Comme le montre cette querelle, si les combinaisons retenues peuvent être l'objet de tensions, par contre le recours au système semble admis par tous les géographes impliqués dans cette controverse ; il apparaît même comme une source de rigueur, une manière de légitimer les faits mobilisés.

\section{Les enjeux du système de Philippe Buache}

Cette unanimité des géographes à l'égard des systèmes ne peut cependant pas être généralisée; leur usage a en effet suscité quelques polémiques. Ainsi, Constantin François de Volney a-t-il condamné I'usage des systèmes auquel Charles de Montesquieu notamment avait eu recours pour décrire des groupes de population à partir de lois générales; et, dans son Voyage en Syrie et en Égypte, il a exalté l'enquête empirique comme fondement de toute connaissance des régularités ${ }^{11}$. À cette exception près cependant, la géographie politique des lumières a plutôt donné lieu à l'écriture de textes qui inventorient les états et leurs habitants, sans manifester le souci de faire apparaître des principes généraux. En revanche, à partir des années 1750, la géographie physique a été plus largement traversée par ces questionnements sur l'usage des systèmes. De manière plus polémique, certains géographes de la deuxième moitié du XVIII ${ }^{\mathrm{e}}$ siècle ont

10 - Ibid., 2-3.

11 - Considérant qu'on ne peut dégager des lois générales qu'à partir des observations, Volney note en effet dans son Voyage en Syrie et en Égypte : "On va même jusqu'à prendre ces faits en preuve d'un paradoxe moral témérairement avancé ; et l'on veut en appuyer ce prétendu axiôme, que les habitans des pays chauds, avilis par tempérament et par caractère, sont destinés par la nature à n'être jamais que les esclaves du despotisme. Mais a-t-on bien examiné si des faits semblables ne sont jamais arrivés dans les climats qu'on veut honorer du privilège exclusif de la liberté ? A-t-on bien observé si les faits généraux dont on s'autorise, ne sont point accompagnés de circonstances et d'accessoires qui en dénaturent les résultats? II en est de la politique comme de la médecine, où des phénomènes isolés jettent dans l'erreur sur les vraies causes du mal. On se presse trop d'établir en règles générales des cas particuliers : ces principes universels qui plaisent tant à l'esprit, ont presque toujours le défaut d'être vagues. Il est si rare que les faits sur lesquels on raisonne, soient exacts ! Et l'observation en est si délicate, que l'on doit souvent craindre d'élever des systêmes sur des bases imaginaires. » Non seulement l'argumentation offre d'évidentes ressemblances avec celle des géographes physiciens que nous évoquerons un peu plus loin, mais le vocabulaire est parfois commun. Volney évoque des "faits généraux », des "phénomènes isolés », il attire l'attention sur le danger $d^{\prime}$ " établir en règles générales des cas particuliers », de " craindre d'élever des systèmes sur des bases imaginaires ». Cependant ces propos, évidemment dirigés contre Montesquieu et sa théorie des climats, permettent surtout à Volney de prendre position dans le champ philosophique. Sa critique n'est pas du même ordre que celle des géographes ; d'ailleurs il existe des linéaments d'un système déterministe climatique du « genre de vie » dans les leçons d'histoire de Volney. (Constantin François de Volney, Voyage en Syrie et en Egypte, pendant les années 1783, 1784 et 1785 [Paris : VollandDesenne, 1787], 178-179.) 
ainsi cherché à mobiliser les systèmes pour établir les principes qui régissent les formes de la terre.

Ainsi, à la manière des systèmes qui rendent compte de la position respective de la Terre et du Soleil comme celui de Ptolémée ou celui de Tycho Brahé, ou à la manière des «théories de la terre » proposées pour exposer la formation de la terre comme celles de Thomas Burnet, de John Woodward, de Gottfried Wilhelm Leibniz, ou de Georges Louis Buffon, certains géographes ont été tentés par les systèmes. C'est fidèle à cet esprit et en cherchant à proposer un cadre général d'explication au relief du globe, que Buache expose, le 15 novembre 1752, à I'Académie royale des sciences, son propre système souvent désigné comme "système des bassins fluviaux ». Dans un mémoire intitulé «Essai de géographie physique ${ }^{12}$ », le géographe présente la structure de cette « espèce de charpente du globe » qu'il a imaginée. Cette notion a déjà été utilisée auparavant: le comte de Marsigli, par exemple, a parlé de "l'ossature de la terre » et, comme beaucoup d'autres, a filé la métaphore anatomique pour décrire les chaînes de montagnes. Cette fois pourtant, le texte de Buache entend prouver l'existence d'une telle structure de manière méthodique.

Pour cela, Buache pose tout d'abord que la connaissance géographique du globe doit commencer par l'identification des montagnes qui, selon lui, ont pour fonction de séparer les bassins fluviaux les uns des autres ; puis, en s'appuyant sur ce postulat, il montre que toute ligne de partage des eaux est obligatoirement matérialisée par une chaîne de montagnes, ce qui lui permet de déduire l'existence de montagnes jusqu'alors méconnues; enfin, il regarde ces chaînes comme une " espèce de charpente », comme "le soutien des différentes parties du globe terrestre »; leur continuité d'un continent à l'autre assurant, à ses yeux, la solidité de l'édifice.

Cette «charpente de la terre » que Buache expose en 1752 a été élaborée peu à peu. La lecture des recueils de l'Académie royale des sciences témoigne de cette genèse graduelle. D'après l'Histoire de I'Académie, c'est en 1737 que Buache dresse la première carte qui cherche à rendre compte des montagnes du globe terrestre. Pourtant, cette carte de la partie de l'Océan vers l'Équateur, comprise entre les continents d'Afrique et d'Amérique n'est présentée à l'Académie qu'en 1745. Rétrospectivement, elle est toutefois regardée comme «la première ouverture $d^{\prime}$ un système général qu'il méditait 13 ». II s'agit en effet d'une

12 - Philippe Buache, Essai de géographie physique où I'on propose des vues générales sur l'espèce de charpente du globe, composée de chaînes de montagnes qui traversent les mers comme les terres; avec quelques considérations particulières sur les différents bassins de la mer intérieure, Mémoires de l'Académie royale des sciences (MARS par la suite) (1752) (Paris, 1754).

13 - Histoire de l'Académie royale des sciences (HARS par la suite) (1752), 117. 
« carte d'une espèce bien singulière puisqu'au lieu de représenter la surface de la mer comme les autres cartes marines, elle en représente au contraire le fond et fait voir que le terrain recouvert par les eaux de I'Océan a ses vallées, ses plaines et ses montagnes et [...] que les chaînes qu'elles forment sont exactement la prolongation de celles que forment les montagnes terrestres ${ }^{14} »$.

Sur cette carte, Buache fait donc disparaître le bleu qui, traditionnellement, sur les cartes enluminées, recouvre de manière uniforme la surface des mers. En procédant ainsi, il est convaincu de révéler la charpente du globe, cette espèce de structure invisible rend compte de la morphologie de la terre. Cette astuce doit donner à voir le fond de la mer et Buache l'exploite encore à plusieurs reprises ensuite, notamment avec la Carte physique de l'Océan qu'il présente à l'Académie le 5 septembre 1744 (voir illustration 6) ${ }^{15}$. Cette carte représente les chaînes de montagnes qui traversent I'océan Atlantique et I'océan Méridional pour relier l'Europe et I'Amérique, mais aussi I'Afrique et I'Amérique, ou encore l'Amérique du Sud et l'Afrique du Sud avec les «terres antarctiques». Comme le suggèrent à la fois le titre complet de la carte et le tracé adopté par Buache, les terres émergées - qu'il s'agisse des îles, des bancs, des roches ou des vigies - apparaissent comme des fragments visibles de cette armature - des sommets émergés - qui prouvent l'existence de ces chaînes de montagnes dissimulées par les eaux. Ainsi, selon Buache, la Sierra Leone est une chaîne de montagnes qui traverse la Guinée, délimite le bassin fluvial du Sénégal, se poursuit au fond de l'Océan pour reparaître au Brésil avec la chaîne de la "Cordelière ». Cette continuité est suggérée sur la carte par la position de l'île de Noronha, située au sud de l'Équateur, mais aussi par la mention textuelle que Buache a insérée sur sa carte pour indiquer qu'à cet endroit se trouvaient des «vigies et roches sous l'eau ». Sur la même carte, on voit la partie méridionale des monts Atlas se prolonger dans I'Océan par les îles Canaries, Madère et les Açores ; cette chaîne reparaît ensuite à l'embouchure du Saint-Laurent avec le «Grand Banc».

Sur cette carte, comme sur toutes celles qui donnent à voir cette hypothétique continuité des chaînes de montagnes terrestres et marines, toutes les îles sont considérées comme le sommet des montagnes marines ; mais Buache ne s'interroge pas sur leur nature, il met bout à bout des montagnes qui sont parfois d'âge et de formation différents ; de plus, le tracé qu'il en donne est souvent inexact; mais il ne se soucie d'aucun de ces aspects, pas plus qu'il ne se préoccupe de la

14 - HARS (1752), 117.

15 - Carte physique de l'Océan où l'on voit les grandes chaînes de montagnes qui traversent les continents d'Europe, d'Afrique et d'Amérique, tous les terreins inclinés vers cette mer et les fleuves qui s'y rendent, avec la continuation de chaînes de montagnes marines, par les isles, les bancs, les roches, ou vigies, par Philippe Buache. Cette carte fut publiée sous le privilège de l'Académie royale des sciences le 4 septembre 1754 ; elle fait également partie des cartes de Buache reprises dans le volume Cartes et tables de la géographie physique ou naturelle, présentées au roi le 15 mai 1757 (Paris, 1757). 
nature des terrains, ou des phénomènes d'érosion. Ainsi, comme le relève Numa Broc, "les montagnes ne sont finalement jamais considérées en elles-mêmes, mais uniquement en tant que limites, barrières ou supports 16 ». Buache superpose à la nature un système qui donne une fonction spécifique à chaque objet physique représenté et qui permet de cartographier des objets qui, jusqu'alors, ne l'étaient pas. Ces nouveaux éléments sont censés donner du sens à la géographie du globe et permettre à la fois de hâter la connaissance des contours de la terre et de passer d'une géographie descriptive à une géographie explicative. Pour cela, Buache estime que la géographie doit s'inspirer des méthodes de la physique ; il néglige cependant les recommandations prudentes formulées notamment dans la préface sur l'utilité des mathématiques et de la physique. Buache semble en particulier ignorer la différence qui est alors énoncée entre la physique systématique et la physique expérimentale. Dans la préface de Fontenelle, publiée en tête de l'Histoire de l'Académie des sciences, on peut lire :

«Aussi l'Académie n'en est-elle encore qu'à faire une ample provision d'observations et de faits bien avérés, qui pourront être un jour les fondemens d'un sistème; car il faut que la phisique sistématique attende à élever des édifices ; que la phisique expérimentale soit en état de lui fournir les matériaux nécessaires ${ }^{17}$. »

Contrairement aux recommandations de Fontenelle qui insiste sur la nécessité de prendre la nature par petites parcelles, Buache utilise quant à lui les chaînes de montagnes marines comme un élément capable de structurer ses déductions géographiques. Elles constituent même le point de départ de son système. Dans ce dispositif, la carte ne consiste pas tant à fixer un état des connaissances géographiques, mais elle apparaît plutôt comme un outil «qui en permet le dépassement ${ }^{18}$ », elle permet de représenter des espaces pour lesquels les observations manquent. Buache donne ainsi à ses lecteurs l'impression que les découvertes futures viendront confirmer les cadres généraux qu'il a posés; et dès qu'il le peut, il rappelle que les découvertes de tel ou tel navigateur corroborent ce qu'il a soupçonné ou déduit. Ainsi, dans le mémoire qu'il présente le 9 août 1752 à l'Académie royale des sciences, pour faire connaître les apports de la relation de I'amiral de Fonte, il note : «Cette relation me frappa beaucoup parce qu'elle fournit à la première esquisse que j'en fis la liaison de l'Asie avec I'Amérique telle que je I'avois soupçonnée auparavant ${ }^{19}$. » Buache s'efforce de montrer, dans des cartes et des essais, la validité de tous ses principes et la pertinence du système qu'il a construit : ce

16 - Numa Broc, La Géographie des philosophes (Paris : Ophrys, 1975), 204.

17 - Bernard Fontenelle, op. cit. in n. 7, XXVII.

18 - Christian Jacob, Quand les cartes réfléchissent, EspacesTemps, Les Cahiers, Penser/figurer : L'espace comme langage dans les sciences sociales, 62-63 (1997), 43.

19 - Philippe Buache, "Considérations géographiques et physiques sur ce que la carte des nouvelles découvertes offre de plus particulier», ms, pochette 9 août 1752 (Arch. de I'Académie royale des sciences), 1. 


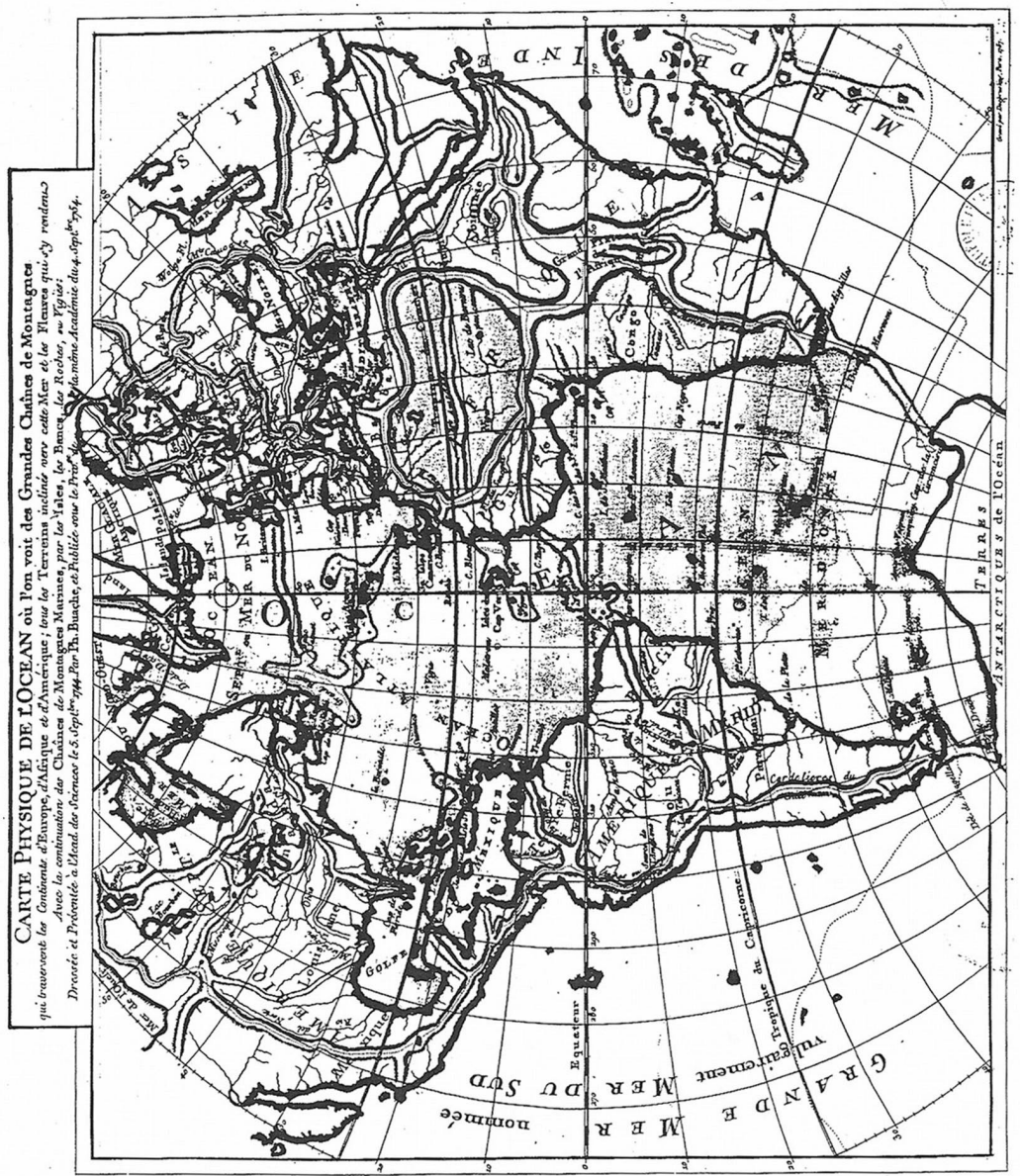

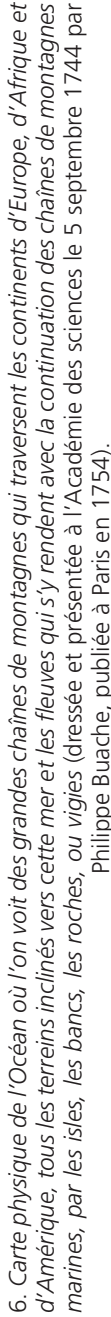


souci prime sur la structuration des connaissances géographiques. $C^{\prime}$ est d'ailleurs un point sur lequel sa méthode diffère fondamentalement de celle de Robert de Vaugondy.

Dans I'Éloge de Buache qu'il prononce en 1773, Jean-Paul Grandjean de Fouchy parle de cette approche suivie par l'adjoint-géographe de I'Académie royale des sciences comme d'une «ingénieuse hypothèse » qui "fut même en quelque sorte prophétique [...] 20 ». II considère la carte de 1744 comme une « esquisse du grand travail qu'il méditait sur la structure du globe terrestre et sur l'arrangement et I'usage des montagnes 21 ». Rétrospectivement, il va, lui aussi, jusqu'à présenter le système comme un moyen d'anticiper les découvertes issues des navigations ${ }^{22}$. En revanche, les membres de l'Académie des sciences qui ont rendu compte des travaux de Buache regardent cette connaissance des océans d'une autre manière. Dans le volume de I'Histoire de l'Académie des sciences consacré à l'année 1745, le travail de Buache est présenté avec plus de circonspection. Le rapporteur détaille les modalités de construction du système, il se montre donc plus précis que Grandjean de Fouchy et explique que la preuve de la continuité des chaînes de montagnes « se tire des basses et des vigies qui se rencontrent sur la ligne qui joint le cap Tagrin et l'embouchure du rio Grande »; il s'appuie donc avant tout sur des éléments du relief visibles qui traversent l'Océan, éléments dont Buache a montré I'importance. Toutefois, le rapporteur ne s'en tient pas là, il poursuit son argumentation en rappelant que Buache a utilisé « une multitude de sondes et d'observations 23 »; sondes et observations dont ni cette carte ni le texte de Buache ne présentent pourtant de traces mais qui, aux yeux des académiciens, apparaissent comme un préalable indispensable à la construction d'une carte. Enfin, à aucun moment ce rapporteur ne regarde cette carte comme prophétique, il considère cependant que de telles cartes des mers pourront être très utiles aux navigateurs :

« Il est aisé d'en apercevoir toute l'importance et de voir combien des cartes pareilles des mers fréquentées seroient utiles, tant par la connoissance des bancs et des roches qu'on doit éviter, que par la lumière qu'elles jeteroient nécessairement sur la direction des marées et des courants dont les plus bizarres phénomènes dépendent peut-être de cette disposition cachée du fond de la mer ${ }^{24}$. »

Face à l'approche spéculative de Buache, les membres de l'Académie des sciences conservent un raisonnement géographique empirique.

20 - Jean-Paul Grandjean de Fouchy, "Éloge de Philippe Buache », HARS, IX, 142.

21 - HARS, IX, 141.

22 - « Nous verrons bientôt qu'elle [C'est-à-dire son « ingénieuse hypothèse »] avait donné lieu à M. Buache de deviner une bonne partie de ce que les recherches faites en Russie par M. de I'Isle l'astronome lui avoient appris sur la partie septentrionale de la mer du sud » (Jean-Paul Grandjean de Fouchy, op. cit. in n. 20,142.)

23 - HARS (1745), 76.

24 - HARS (1745), 77.

Revue d'histoire des sciences | Tome 59-1 | janvier-juin 2006 
On retrouve bien ici deux manières différentes de tirer parti d'un système: Buache regarde les observations comme un moyen de compléter a posteriori la structure suivant laquelle il appréhende la structure du globe - pour lui c'est le système qui "fait science »-, alors que les membres de l'Académie des sciences entendent partir des observations pour dégager des régularités et produire ainsi des connaissances sûres. Buache raisonne donc par conjectures et analogies; autant de principes qui semblent contradictoires avec ceux défendus par les membres de l'Académie royale des sciences; pourtant, malgré ces défauts ${ }^{25}$, son système est plutôt encouragé par les membres de cette prestigieuse institution ; probablement parce qu'il rompt avec l'approche strictement descriptive de la géographie moderne. Ainsi, dans I'ensemble, les académiciens qui ont rapporté les travaux de Buache se sont-ils félicités de cette manière d'aller « vers I'entière connoissance du globe terrestre 26 ", mais aussi de cette " façon de considérer notre globe [qui] ouvre une nouvelle carrière à la géographie 27 ». Cette méthode géographique que propose Buache semble en effet rompre avec le passé :

"Cette façon si naturelle de considérer le globe terrestre avoit cependant été absolument ignorée ou négligée avant $\mathrm{M}$. Buache : il en a ouvert la première idée, il en a fait voir les avantages et on lui devra toujours cette espèce de géographie \& la nouvelle route qu'il a ouverte aux amateurs de cette science ${ }^{28}$. »

Toutefois, pour les académiciens, ce système qu'ils disent « conforme aux vues de la saine physique 29 " n'est pas définitif, il doit au contraire être complété voire réajusté en fonction des observations ultérieures ${ }^{30}$. On retrouve ici la trace des recommandations qui étaient déjà formulées dans la "Préface sur l'utilité des mathématiques et de la physique » de Fontenelle; on peut y lire notamment :

« Jusqu'à présent l'Académie des sciences ne prend la Nature que par petites parcelles. Nul sistême général, de peur de tomber dans l'inconvénient des sistêmes précipités dont l'impatience de l'esprit humain ne s'accommode que trop bien, \& qui étant une fois établis, s'opposent aux vérités qui surviennent. Aujourd'hui on s'assure d'un fait, demain d'un autre qui n'y a nul rapport. On ne laisse pas de hazarder des conjectures sur les causes, mais ce sont des conjectures ${ }^{31}$. »

25 - Dans les rapports rédigés par Pierre Bouguer, Montigny et Louis-Guillaume Le Monnier, on décèle sans peine la méfiance que ressentent ces académiciens à l'égard des « soupçons » et des " conjectures » sur lesquels repose ce nouveau système. Aux Archives de l'Académie des sciences, on trouvera le rapport de Bouguer et Montigny dans la pochette de la séance du 4-7 juillet 1753, le rapport de Bouguer et Le Monnier dans la pochette de la séance du 24 juillet 1754, le rapport de Bouguer dans la pochette de la séance du 4 août 1754.

26 - HARS (1750), 152.

27 - HARS (1752), 123

28 - HARS (1756), 111.

29 - HARS (1757), 142.

30 - HARS (1745), 77 ; HARS, (1752), 123 ; HARS (1753), 271

31 - Fontenelle, op. cit. in n. 7, XXVIII-XXIX. 
De telles mises en garde furent réaffirmées au milieu du $X V I^{\mathrm{e}}{ }^{\mathrm{e}}$ siècle. Ainsi, dans I'Encyclopédie, la "vraie physique » est-elle présentée comme «celle qui s'appuie sur les expériences, \& qui les éclaire par des raisonnemens exacts \& précis, \& non pas par des explications vagues ». Or, cette méthode ne correspond manifestement pas à celle que Buache a suivie. Si l' "Essai de géographie physique» de Buache ne relève pas de la vraie physique, on peut, de la même manière, considérer qu'il n'est pas un "vrai système ». Dans l'article "système » qu'Étienne Bonnot de Condillac a rédigé pour l'Encyclopédie, les « vrais systèmes » sont définis comme ceux fondés seulement «sur des faits bien prouvés »; en cela, ils s'opposent aux "systèmes abstraits » et aux " hypothèses » " qui n'ont que des suppositions pour fondement ». C'est plutôt de cette dernière catégorie que semble donc relever le système de Buache. On discerne en effet dans ses travaux une volonté d'expliquer la nature en ayant recours à quelques principes abstraits, mais sans disposer d'un nombre suffisant d'observations. Comme l'a montré Broc, chez Buache, "la synthèse précède les faits 32 ». Pourtant, ce système a été enseigné et utilisé jusqu'au début du XIX ${ }^{\mathrm{e}}$ siècle, notamment par des ingénieurs topographes. Broc a évoqué cette "faveur extraordinaire 33 » du système de Buache, et plus récemment, Valeria Pansini ${ }^{34}$ a montré combien ses travaux ont permis de définir des principes d'observation qui, d'une part, aidaient à comprendre le tout ou à l'imaginer et qui, d'autre part, donnaient le moyen d'en construire une représentation.

\section{L'emprise des systèmes dans les premiers travaux de Nicolas Desmarest}

Malgré cette discordance entre les attentes des philosophes et les manières de faire de Buache, cet usage des systèmes ne peut être regardé comme une pratique isolée. En effet, en 1751, Nicolas Desmarest rédige une Dissertation sur l'ancienne jonction de l'Angleterre à la France qu'il présente au concours de l'académie d'Amiens. Deux années plus tard, ce texte qui a remporté le concours académique est imprimé. Il permet de mesurer le statut très ambigu des systèmes à ce moment où les théories de la terre ne convainquent plus vraiment mais où, cependant, on ne s'est pas encore résolu à renoncer aux explications ${ }^{35}$. Ainsi, au début de la deuxième partie de sa dissertation, Desmarest trahit bien cette fascination durable qu'exercent les théories de la terre sur les savants qui tentent de la décrire et aspirent plus encore à en expliquer les formes; il note :

32 - Broc, op. cit. in n. 16, 181.

33 - Numa Broc, Les Montagnes vues par les géographes et les naturalistes de langue française au XVIII ${ }^{e}$ siècle (Paris : CTHS- Bibliothèque nationale, 1969), 61.

34 - Valeria Pansini, "L'œil du topographe et la science de la guerre : Travail scientifique et perception militaire (1760-1820) », thèse nouveau régime (EHESS, Paris, 2002).

35 - Kenneth Taylor, Les lois naturelles dans la géologie du XVIII ${ }^{\mathrm{e}}$ siècle, Travaux du COFRHIGEO (Comité français d'histoire de la géologie) (Paris), ser. 3, t. 2 (1988), 1-28. 
«Tout se tient dans le méchanisme de l'univers. Les principales opérations de la nature en grand, influent sur les effets subalternes. C'est de la connoissance bien développée de ces agens généraux, que dépendent le dénouement et l'intelligence des phénomènes particuliers. Notre globe a éprouvé à sa surface des changemens et des révolutions plus ou moins étendus. Les preuves en sont répandues partout ; \& quiconque en dispute l'existence, foule peut-être aux pieds les monumens qui attestent ces altérations. La cause qui a produit ces effets doit être prise dans la méchanique générale à laquelle les grands phénomènes de notre globe sont assujettis ${ }^{36}$. »

Certes, en évoquant "les preuves » de changements qu'a connus le globe, Desmarest suggère la place qu'il entend laisser à l'observation, et d'ailleurs au début de son texte il affirme qu'il ne veut pas se laisser " entraîner par l'amour du merveilleux, qui engage trop souvent à étayer des récits chimériques \& hazardés, sur des fondemens aussi ruineux; à soutenir \& à réaliser des événemens dont l'ignorance ou I'imagination ont fait tous les frais 37 ».

Cependant, au travers de cette entreprise, Desmarest n'entend pas décrire un espace particulier - où il ne s'est jamais rendu. Son ambition consiste au contraire à utiliser le canal de la Manche comme un indice qui prend place à l'intérieur d'un projet plus vaste ${ }^{38}$. Ces précautions rhétoriques sur les dangers de l'imagination ne permettent donc pas de conclure que Desmarest se démarque radicalement des auteurs des théories de la terre, ni des travaux de Buache; le géographe est d'ailleurs omniprésent dans cette dissertation.

Tout d'abord, il est l'auteur de la carte de la Manche que Desmarest joint à son texte. En effet, l'année précédente, dans son "Essai de géographie physique », Buache a déjà inséré une carte de la Manche pour consolider sa démonstration et montrer la pertinence de la structure du globe qu'il décrivait. La planche réalisée par Buache présente donc une carte et une coupe (voir illustration 7). La coupe est censée donner à voir ce que la carte a du mal à dévoiler, c'est-à-dire ici les variations du niveau du fond de la mer. Buache a en effet imaginé un système de correspondance entre la coupe et la carte. Tout d'abord, il a représenté sur la carte une ligne graduée qui coupe en deux le canal de la Manche d'ouest en est, ligne qui est censée montrer l'espace représenté sur la coupe ; puis il a reporté sur la carte et sur la coupe des repères ponctuels identifiés par les huit premières lettres de I'alphabet qui facilitent aussi le passage d'un document à l'autre. Cette correspondance de la coupe et de la carte permet à Buache de montrer

36 - Nicolas Desmarest, Dissertation sur l'ancienne jonction de l'Angleterre à la France, qui a remporté le prix, au jugement de l'Académie des sciences, belles-lettres et arts d'Amiens, en I'année 1751 ; avec des plans et des cartes topographiques (Amiens : Veuve Godart, 1753), 69.

37 - Desmarest, op. cit. in n. 36, 3-4.

38 - Kenneth L. Taylor, La genèse d'un naturaliste : Desmarest, la lecture et la nature, in De la géologie à son histoire (Paris : CTHS, 1997), 61-74. 
que la profondeur du canal de la Manche passe d'environ 100 brasses entre les îles Sorlingues et Ouessant à 40 brasses au large de l'île de Wight pour se réduire à moins de 20 brasses dans le Pas-de-Calais. Pour délimiter les sept « lits » qu'il identifie dans le canal de la Manche en fonction de leur profondeur (39), Buache dit s'appuyer sur des mesures du niveau de la mer faites grâce à des sondes. Ces données lui ont permis non seulement de tracer sa coupe, mais aussi de dessiner des isobathes, courbes de niveau marines. Comme c'est souvent le cas quand il évoque l'origine des mesures qu'il utilise, Buache se montre aussi très allusif dans le mémoire qui accompagne cette carte. II se contente en effet de mentionner qu'il a réuni « des sondes que les navigateurs ont observées », sans préciser à quel moment ces mesures ont été effectuées, sans indiquer la liste de ces mesures. Or, ce sont ces mêmes données qu'il a transmises à Desmarest et que celui-ci a utilisées pour étayer sa démonstration. II note ainsi :

«Monsieur Buache si zélé pour le progrès de la géographie physique, \& qui a des vues si étendues sur toute l'économie et la disposition des inégalités de notre globe, a bien voulu me faire part d'un détail d'opérations fort intéressantes sur toute la Manche et la mer d'Allemagne. Il a réuni et combiné toutes les observations avec les plus anciennes, \& on connoît son exactitude et sa sagacité à tirer parti de tous les faits détachés, pour les rapprocher heureusement, \& former un tout et un ensemble qui les mette dans leur juste point de vue ${ }^{40}$. »

Pourtant, ni le texte de Buache, ni celui de Desmarest ne permettent de situer l'origine de ces observations, et, une fois encore, ces mentions font surtout penser à des précautions rhétoriques qui mobilisent le langage de I'observation; il est en effet difficile de voir ici la mise en œuvre d'un protocole d'expérimentation rigoureux. À cet égard d'ailleurs, les essais des deux hommes se ressemblent et c'est à plusieurs reprises que Desmarest fait allusion au système de Buache dans sa dissertation. II ne condamne pas le raisonnement systématique du premier géographe du roi, au contraire il semble plutôt utiliser l'approbation de l'Académie des sciences comme une caution à sa propre démarche. Si la forme du raisonnement est identique, les principes mis en œuvre sont cependant très différents et Desmarest insiste sur cet aspect pour montrer la spécificité de son approche. II rappelle le principe du système de Buache - les chaînes de montagnes terrestres se poursuivent dans la mer - et ajoute :

« Cette idée de jonction sous les eaux est bien différente de la jonction au-dessus de l'eau que j'ai adoptée dans ma dissertation et par laquelle je conçois les rivages actuels anciennement réunis et formant au lieu du canal un terrain de plein pied \& un seul continent. J'ai cru devoir caractériser avec précision ces deux manières de considérer la jonction de I'Angleterre avec la France, de peur que sous les mêmes termes, on

39 - MARS (1752), 412-413.

40 - Desmarest, op. cit. in n. 36, 60-61.

Revue d'histoire des sciences | Tome 59-1 | janvier-juin 2006 


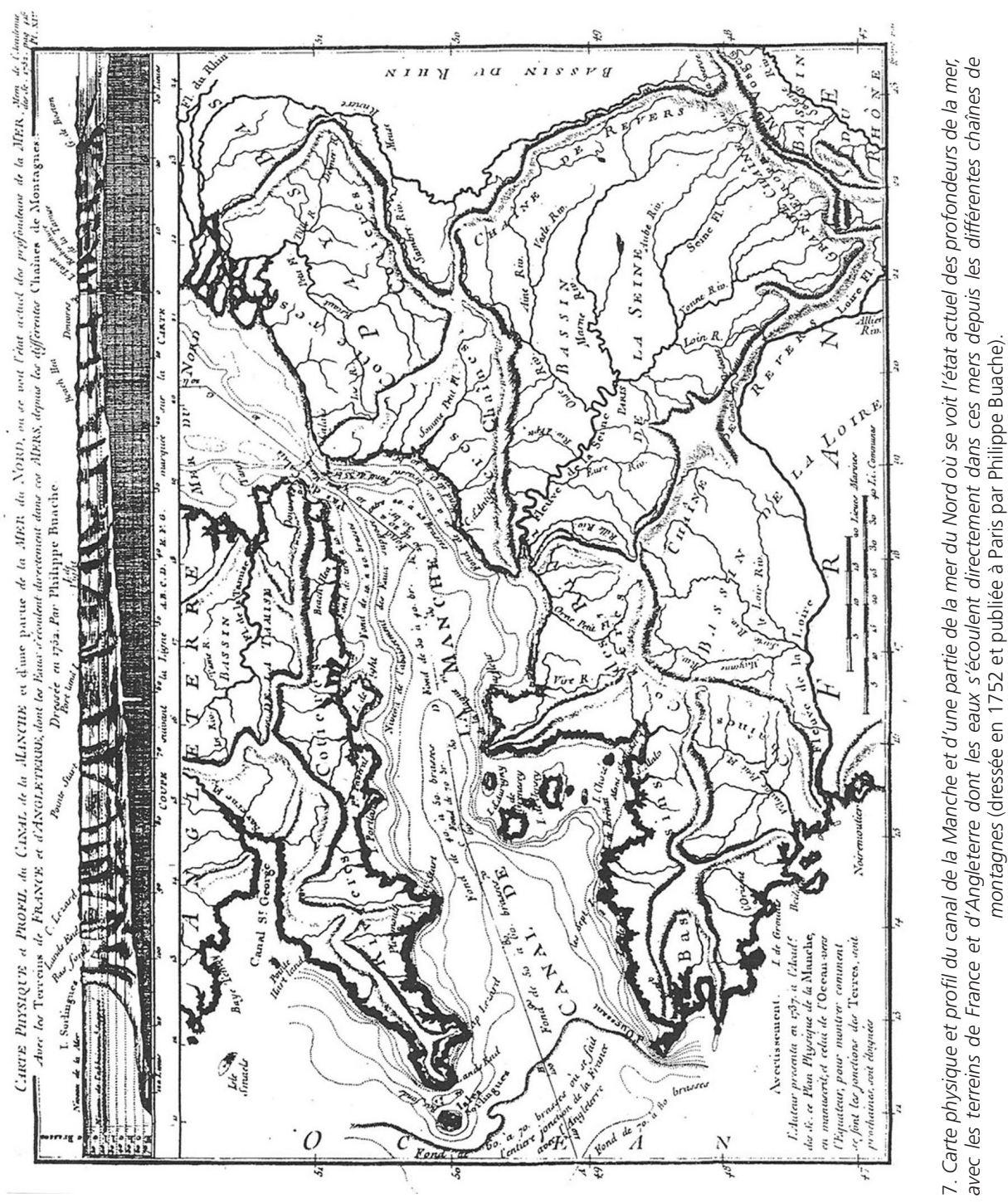


ne les confondît. Ainsi, M. Buache a été seulement occupé à représenter \& à décrire l'état actuel des lieux, sans vouloir jeter ses regards sur I'ancienne disposition, au lieu que je pars de l'état actuel pour remonter à I'ancienne disposition \& la conclure ${ }^{41}$. »

Certes, les démarches sont différentes, cependant quoi qu'il en dise, le mode de raisonnement développé par Desmarest est assez semblable à celui de Buache. Comme lui, il se contente de constater l'adéquation des observations dont il dispose avec son système. II cherche pour sa part à prouver que I'Angleterre et la France ne faisaient qu'un seul continent en montrant qu'un exhaussement du niveau de la mer a transformé en détroit l'isthme qui séparait le Boulonnais de la côte anglaise. Il conclut ainsi que si le niveau de l'Océan s'abaissait de 20 à 23 brasses, le détroit redeviendrait isthme ${ }^{42}$. Ce ne sont cependant pas les observations qui déterminent l'analyse de Desmarest et qui lui permettent d'imposer cette conclusion. Dans le corps de son texte, il souligne ainsi : "Les observations les plus modernes sont conformes à celles qui m'ont guidé dans mes raisonnemens sur la disposition du terrain ${ }^{43}$. " Comme Buache, Desmarest développe donc un mauvais raisonnement inductif ; il se contente de vérifier la validité des observations qu'il a réunies en les confrontant aux principes qu'il a posés au préalable.

\section{Les systèmes mis à l'épreuve des faits}

Quelques années plus tard pourtant, dans l'article « géographie physique » qu'il rédige pour le septième volume de l'Encyclopédie (1757), Nicolas Desmarest condamne, à son tour, les systèmes qui ne reposent pas sur l'observation et qui risquent d'agir comme des prismes déformants pour les observations à venir ${ }^{44}$. Il oppose ainsi deux manières de faire : d'une part la procédure fondée sur l'observation et la généralisation prudente, d'autre part la méthode hypothético-déductive où I'on postule a priori avant de vérifier les conséquences formulées par déduction. Desmarest se place du côté des défenseurs de l'empirisme, il affirme ainsi :

« Nous croyons aussi que l'observateur doit être en garde contre toute prévention, toutes vûes fixes \& dépendantes d'un système déjà concerté : car dans ce cas, on interprete les faits suivant ce plan ; on

\footnotetext{
41 - Ibid., IV-V.

42 - François de Dainville, De la profondeur à l'altitude : des origines marines de l'expression cartographique du relief terrestre par cotes et courbes de niveau, in La Cartographie reflet de I'histoire : Recueil d'articles de François de Dainville présenté par Michel Mollat du Jourdin (Genève-Paris : Slatkine, 1986), 441-455.

43 - Desmarest, op. cit. in n. 36, 60.

44 - Isabelle Laboulais, Voir, combiner et décrire: La géographie physique selon Nicolas Desmarest, Revue d'histoire moderne et contemporaine, LI/2 (2004), 38-57.
} 
glisse sur les circonstances qui sont peu compatibles avec les principes favoris, \& I'on étend au contraire celles qui paroissent y convenir ${ }^{45}$. »

Et il ajoute :

«On se laisse souvent séduire dans le cours de ses observations, ou bien par négligence, ou bien par une prévention de système; en conséquence on a la présomption de voir au-delà de ce que la nature nous montre, ou bien l'on craint d'appercevoir tout ce qu'elle peut nous découvrir. D'après cette illusion, on imagine de la ressemblance entre les objets les plus dissemblables, de la régularité \& de l'ordre au milieu de la confusion ${ }^{46}$. »

Si radical que puisse paraître ce changement de ton, on doit retenir qu'exprimer ainsi sa réserve à l'égard des systèmes devient à partir de la fin des années 1750 un véritable topos.

Il faut en effet rappeler qu'en 1749 Condillac a publié son Traité des systèmes, où l'on en démelle les inconvénients et les avantages. Cet ouvrage, dont le contenu est d'ailleurs repris par Condillac lui-même dans l'article "Système » de l'Encyclopédie, propose de distinguer trois sortes de systèmes : des «maximes générales et abstraites » que Condillac nomme des " systèmes abstraits », " des suppositions qu'on imagine pour expliquer les choses dont on ne saurait d'ailleurs rendre raison » qu'il désigne comme des «hypothèses », enfin des "vrais systèmes » qui sont "fondés sur des principes constatés par l'expérience $^{47} »$. Dès que ces différences sont énoncées et que l'on admet que seuls les « faits constatés » peuvent constituer les « seuls principes des sciences », I'universalité des systèmes est ensuite posée. Au chapitre XIV de son traité, Condillac note :

« II n'y a point de science ni d'art où l'on ne puisse faire des systèmes : mais dans les uns, on se propose de rendre raison des effets; dans les autres de les préparer \& de les faire naître. Le premier objet est celui de la physique; le second est celui de la politique. Il y a des sciences qui ont l'un et l'autre, telles sont la chimie et la médecine ${ }^{48}$. »

Dans I'article de I'Encyclopédie, Condillac reprend ce même passage qui affirme qu'aucune science ne semble a priori empêchée d'utiliser les systèmes. Il ajoute même dans le dernier chapitre de son ouvrage : « [...] une science bien traitée, est un système bien fait 49 ».

Les naturalistes ont repris progressivement à leur compte ces arguments et ont condamné l'esprit de système en général. La charpente du

\footnotetext{
45 - Nicolas Desmarest, Géographie physique, Encyclopédie ou dictionnaire raisonné des sciences, des arts et des métiers (Paris, 1757), 615.

46 - Ibid., 45, 617.

47 - Étienne Bonnot de Condillac, Traité des systèmes, où l'on en démelle les inconvénients et les avantages (Paris : Fayard, 1991 [1 $1^{\text {re }}$ éd. : 1749]).

48 - Ibid., 247.

49 - Ibid., 266.
} 
globe imaginée par Buache est l'un de ces systèmes abstraits qui est alors particulièrement visé. Ainsi, dans le premier volume de son Dictionnaire de géographie physique qu'il rédige en 1795 pour l'Encyclopédie méthodique de Panckoucke, lorsque Desmarest examine les principales théories de la terre, il consacre une notice à Buache et c'est en s'appuyant sur des éléments de reliefs bien connus qu'il se moque de la méthode de ce dernier et disqualifie les conclusions auxquelles il est parvenu :

« Où trouver par exemple une chaîne continue, comme Buache l'a tracée, depuis les sources du Rhin, du Rhône et du Pô, jusqu'aux sources de la Loire \& de l'Allier. Quelles vastes \& grandes vallées ne voit-on pas dans ce trajet ? De même sur quels fondemens établit-on la réunion des deux massifs d'où sortent les sources de la Loire \& de I'Allier avec la branche des arrêtes qu'on suppose prolongées depuis le Velay et l'Auvergne, jusqu'au Pas-de-Calais par les Vôges ? En suivant aussi la trace de la chaîne prétendue, qui des Vôges va se rendre au même Pas-de-Calais, où trouve-t-on une arrête marquée \& élevée au dessus des terreins circonvoisins au milieu des plaines et des collines de la Lorraine, de la Champagne \& de la Picardie, \& une même nature de pierres anciennes \& primitives au milieu de toutes ces couches horizontales de pierres, de sables, de pierres coquillières, de marne \& de craie, qui règnent dans tout le trajet que parcourent ces arrêtes 50 ? "

Se démarquer des méthodes de Buache constitue pour Desmarest une étape primordiale dans son entreprise de définition de la géographie physique. En effet, pour légitimer le champ auquel il entend consacrer ses travaux, il doit mettre en exergue ce qui le différencie de l'esprit de système. II note ainsi dans le premier volume de son dictionnaire :

« Ce sont cependant toutes ces suppositions erronées dont on a fait un corps de doctrine sous le titre de Géographie physique, \& qui, dans ce siècle éclairé, ont été adoptées sans discussion par des géographes ignorans. Cependant il auroit été facile de reconnoître que ces travaux géographiques, rédigés sur des hypothèses illusoires, péchoient par des parties essentielles, l'histoire naturelle \& l'exactitude des positions \& des formes du terrain ${ }^{51}$.»

Desmarest condamne ici les «géographes ignorans », il entend de la sorte marquer tout ce qui le sépare de ces derniers. Dans ses propres travaux de géographie physique, Desmarest ne se désigne jamais comme un géographe mais plutôt comme un physicien ou comme un naturaliste; I'identité et la légitimité de ces deux figures de savants reposent alors sur l'expérience et l'observation. Buache en revanche est associé à ces " géographes ignorans » sans être cependant la seule cible des détracteurs des systèmes. Dans son Dictionnaire de géographie physique, Desmarest formule des critiques similaires à l'encontre

50 - Nicolas Desmarest, Buache, Encyclopédie méthodique : Géographie physique, t. I (Paris : Agasse, an III), 70.

51 - Ibid., 804.

Revue d'histoire des sciences | Tome 59-1 | janvier-juin 2006 
de Jean-Étienne Guettard (1715-1786). Celui-ci est présenté comme ayant construit son système des bandes sablonneuses, marneuses et schisteuses ou métalliques à partir d'observations fragmentaires, puis comme l'ayant généralisé aveuglément.

Plus que les systèmes eux-mêmes, $c^{\prime}$ est la hâte de certains savants à les utiliser qui est dénoncée par Desmarest. Ainsi, dans I'article « Géographie physique » de l'Encyclopédie, comme dans le Dictionnaire de la méthodique, Desmarest déplore l'esprit de système sous-jacent, esprit de système qui, selon lui, fausse l'observation; il condamne ainsi la démarche qui consiste à ajuster les faits aux conjectures posées comme préalables à I'observation. Dans l'article "Fontaine» de I'Encyclopédie, il désigne même les "systèmes » comme des « inductions imaginaires». En revanche, Desmarest semble approuver leur utilisation dès lors que les systèmes font office de "plan méthodique où l'on présente les faits avérés \& constans, \& où on les rapproche pour tirer de leur combinaison des résultats généraux [...] » (définition qui se rapproche assez de l'acception de ce mot chez les cartographes). $C^{\prime}$ est d'ailleurs par ce biais qu'il parvient à nuancer ses propos dans la notice qu'il consacre à Buache, il note ainsi :

"Quoique sa doctrine sur les grandes chaînes de montagnes qu'il suppose traverser sans interruption les continens \& les bassins des mers, \& qu'il considère comme la charpente du globe, ne soit appuyée d'aucune preuve solide, cependant en réduisant ces trois objets à ce qu'ils sont dans la nature, on ne peut disconvenir que la correspondance des fleuves avec les montagnes d'où ils sortent, \& avec les mers où ils se rendent ne soit très utile à l'étude de la géographie-physique, sur-tout lorsque ces objets sont dans un rapprochement convenable et point forcé ${ }^{52}$. »

S'il condamne donc la méthode de Buache et ses « idées hypothétiques ", Desmarest propose cependant de continuer à suivre les points de partage des eaux courantes à la surface du globe, il recommande d'étudier et de décrire les bassins des fleuves et des rivières. Il parle même des grands " systèmes » de distribution des eaux courantes à la surface de toutes les parties de nos continents, mais ce terme ne désigne plus cette fois un schéma explicatif, il renvoie au contraire à I'agencement des phénomènes.

À la fin des années 1750, une différence assez nette semble être introduite entre l'esprit de système et l'esprit systématique : la stérilité du premier est reconnue, la richesse du second s'impose progressivement. Comme nombre de ses contemporains, Desmarest considère en effet que la connaissance de la terre doit être fondée sur la collecte des faits, sans pour autant faire de celle-ci la finalité de son entreprise. Plus qu'à l'accumulation, il aspire donc à la recherche des « régularités observables ", puis à la combinaison de ces éléments pour parvenir à 
I'explication des phénomènes. Telle est, selon lui, l'ambition de la " géographie-physique 53 », dont il définit la méthode ainsi :

"On part de ces principes, comme d'un point lumineux, pour éclaircir de nouveau certains sujets par l'analogie; \& en conséquence de la régularité des opérations de la nature, on en voit naître de nouveaux faits qui se rangent eux-mêmes en ordre de système. Ces principes sont pour nous les lois de la nature, sous l'empire desquelles nous soûmettons tous les phénomenes subalternes; étant comme le mot de l'énigme, ils offrent dans une précision lumineuse plus de jeu \& de facilité à l'esprit observateur, pour étendre ses connoissances. Enfin ils ont cet avantage très-important, de nous détromper sur une infinité de faits défigurés ou absolument faux ; ces faits disparoîtront ou se rectifieront à leur lumiere, comme il est facile de suppléer une faute d'impression, lorsqu'on a le sens de la chose ${ }^{54}$.»

À ses yeux, la " géographie-physique » doit donc s'appuyer sur une épistémologie empiriste tout en ayant pour objectif de reconnaître les régularités naturelles. Pourtant, tout en cherchant à concilier description et explication, le géographe-physicien ne doit pas chercher d'explications exhaustives ni de principes de causalité absolus. L'influence de la physique newtonienne et de la philosophie naturelle semble déterminante dans la conception de la "géographiephysique » que développe Desmarest. Cependant, plus que Buache, il est aussi influencé par l'histoire naturelle descriptive. Cette influence détermine d'ailleurs sa décision d'exclure les travaux de Buache du champ de la géographie-physique; Desmarest se montre à cet égard catégorique: " [...] ainsi la charpente du globe telle qu'elle a été indiquée \& figurée sur les cartes de Buache, ne peut être considérée comme un grand fait qui appartienne à la géographie-physique ${ }^{55}$. » Pour Desmarest, elle résulte en effet de conclusions hâtives, procédé qu'il réprouve absolument.

À la fin du XVIII ${ }^{e}$ siècle, l'abus des systèmes est donc capable de disqualifier un savoir et le discours sur le rôle des systèmes dans la construction des connaissances semble capable de légitimer ou de marginaliser les résultats produits. C'est ce qu'affirme Jean-Louis Alibert lorsqu'il publie, en 1797, dans le Magasin encyclopédique, un texte intitulé "Quelques réflexions sur la valeur des systèmes dans l'étude des sciences 56 ». Ce texte est conçu comme une mise au point générale et accessible consacrée à la notion ; grâce à lui, Alibert entend bien mettre un terme à la confusion qui entoure l'usage

53 - À partir de 1795, date de la parution du premier volume du Dictionnaire de géographie physique que Desmarest publie pour la série de l'Encyclopédie méthodique, il réunit systématiquement les deux termes de l'expression par un trait d'union: "Géographiephysique ».

54 - Nicolas Desmarest, op. cit. in n. 50, 802.

55 - Desmarest, op. cit. in n. 50, 71.

56 - Jean-Louis Alibert, Quelques réflexions sur la valeur des systèmes dans l'étude des sciences, Magasin encyclopédique, $3^{\mathrm{e}}$ année, t. IV (Paris : Fuchs), 1797, 460-472. 
polysémique de ce mot. Au début de son texte, il note en effet : « On a tour-à-tour vanté ou déprécié les systèmes ; on les a regardés tantôt comme le dernier perfectionnement de nos connoissances, tantôt comme une entrave pour le génie, ou comme une barrière insurmontable opposée aux progrès de l'esprit humain ${ }^{57}$. »

À condition de multiplier les expériences et de se méfier de l'imagination, Alibert regarde les systèmes comme "la seule méthode de l'esprit, la seule manière dont il puisse sortir de l'ignorance et se dégager de l'erreur 58 ». Il entreprend ensuite d'établir une distinction entre les "vrais systèmes [qui] ont tout fait dans les sciences» et les " faux systèmes [qui] ont tout gâté 59 ». Dans cette dernière catégorie, il range bien sûr tous les systèmes qui relèvent de la métaphysique, mais également ceux qui se rapportent à la physique. Dans sa conclusion, Alibert affirme: "Quand nous disons qu'il faut proscrire les systèmes, nous voulons parler de ces collections incomplètes d'idées qui ne font connoître qu'une face de l'objet, et d'après lesquelles cependant on veut juger et expliquer l'objet entier ${ }^{60}$. » II transforme la fin de son article en un plaidoyer pour la physique expérimentale :

«Observez, classez vos observations, et ne généralisez que lorsque vous y serez conduit par les plus frappantes analogies. Hommes de toutes les sciences, ralliez-vous autour de la philosophie expérimentale ! Elle a ses sources dans la nature; elle ressemble, comme l'a dit Bacon, à ces fleuves intarissables qui grossissent continuellement leur cours ${ }^{61}$.»

Difficile de ne pas trouver ici un écho aux discours qui ont accompagné les tentatives de définition des méthodes de la "géographiephysique » proposées par Desmarest.

Ramenées à l'échelle de la communauté savante, de telles positions semblent convenues. Elles reproduisent la condamnation philosophique des systèmes qui sont au Siècle des lumières couramment associés à des synthèses prématurées, à des déductions mal étayées. Elles sont à la fois conformes aux principes avancés par Condillac et aux idées reprises par Alibert. Cependant, à l'intérieur de la production géographique, de tels discours paraissent plutôt isolés ; ils émanent néanmoins de savants qui ont réfléchi aux manières de faire passer la géographie d'une ambition descriptive à une ambition explicative. S'y arrêter permet de suggérer que la géographie des lumières ne peut

57 - Ibid., 460.

58 - Ibid., 464-465.

59 - Ibid., 465.

60 - Alibert, op. cit. in n. 56, 470.

61 - Ibid., 471-472. 
certainement pas être réduite à un « vide », ni considérée comme une discipline en creux. L'un des enjeux de l'histoire de la géographie française au moment de l'émergence des sciences de l'homme consiste aujourd'hui à exhumer ces discours qui, même s'ils se sont finalement rattachés à d'autres champs disciplinaires, sont bel et bien partis de la géographie ${ }^{62}$.

62 - Hélène Blais, Isabelle Laboulais (sous la dir. de), Géographies plurielles : Les sciences géographiques au moment de l'émergence des sciences humaines (1750-1850) (Paris : L'Harmattan, 2006), " Histoire des sciences humaines». 

- 